\title{
تأثير إضافة نواتج مخلفات معامل الألمنيوم على معامل التوصيل الحراري للخرسانة
}

\author{
محمد قادر البياتي"، علي حسين حميد* *"، أسل تحسين حسين **

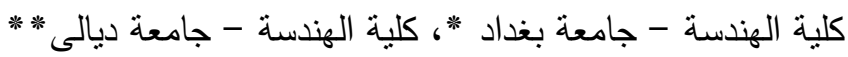

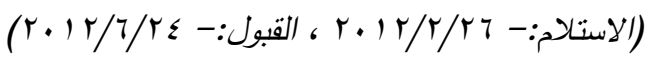

الخلاصة

هذا البحث تتاول تأثثر اضافة الياف الالمنيوم المأخوذة من مخلفات معامل الالمنيوم على خواص الخلطة الخرسانية . الدراسة شملت استخدام خلطة مرجعية (M0) بدون مضاف الالمنيوم لغرض المقارنـة مع باقي الخلطات التي تحتوي

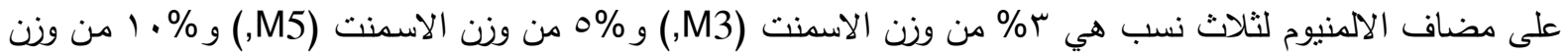
الاسمنت (M10) وبالتحديد معامل التوصيل الحراري للخلطة الخرسانية ( K) بالاعتماد على المعادلة المعتمدة من قبل كود ، أجريت فحوصـات مختبريـة(قابلية النشغيل،الكثافة ،مقاومـة الانضغاط ) لمكعبات الخلطات (ACl 122R-2002) الخرسانية (M10,M5,M3,M0 ) بابعاد (150*150*150 ) ملم ولثلاثة أعمار (28,14,7 ) يوم ـ لبيان مدى تأثر قابلية التشغيل والكثافة و مقاومـة الانضغاط بالمضاف بتقدم عمر النموذجوتاثير ذلك على معامل النتوصيل الحراري للخرسانة. من خلال النتائج التي تم التوصل اليها من الفحوصات وجد ان الكثافة قلت بمقدار (7\%-20\%) مع زيادة نسبة المضاف ومعامل التوصيل الحراري قل بزيـادة نسبة مضاف اليـاف الالمنيوم بحدود ( 17\%-45\%) لارتباطـهـ بالكثافة وكذلك مقاومة الانضغاط نقل باضافة الياف الالمنيوم بحدود (30\%-54\% ) وذلك بسب حصول تفاعل لالياف الالمنيوم مع مركبات الاسمنت الذي يسبب توليد فقاعات غازية وبالتالي حدوث فجوات (خرسانة مهواة) مما يؤدي الى لى حدوث نقصان بالكثافة و مقاومة الانضغاط. مفاتيح الكلمات : معامل التوصيل الحراري ، خرسانة مهواة ، مضاف الألمنيوم ، مقاومة الانضغاط ، الكثافة.

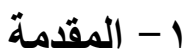

شهد قطاع البناء تطوراً هائلاً في مجال مواد البناء ومنها الخرسانة التي تتميز بسهولة العمل بها وقدرة تحملها العالية ولكن صاحب تلك المواد بعض السلبيات المرتبطة بخصائصها ، فالخرسانة لها خاصية التوصيل السريع للحرارة

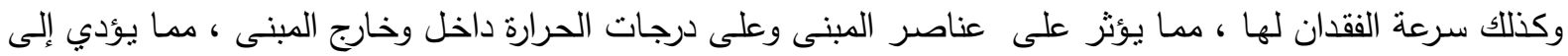

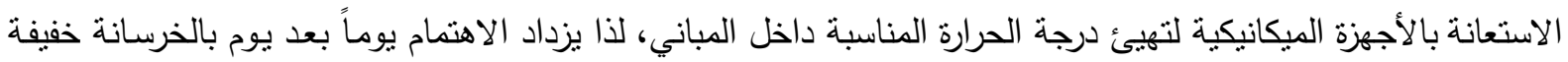
الوزن (Lightweight Concrete) في جميع انحاء العالم وذلك لاسباب اقتصادية بالدرجة الاولى ، فالخرسانة خفيفة

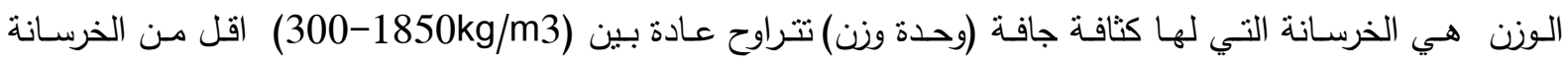

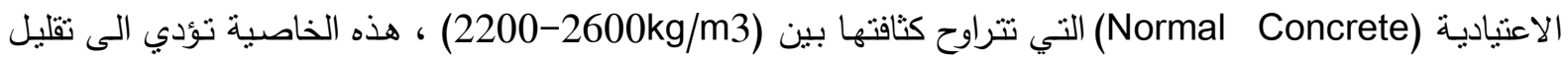
الحمل الكلي المؤثر على المنشأ مها يساعد على استعمال مقاطع انشائية واسس ذات مقاس اصغر ، كما ان القوالب لتردين 
ستتحمل ضغطاً اقل ، وأوزان المواد عند النقل والمناولة اقل، مما يعني إنتاجية أعلى ، كذلك تمتاز الخرسانة خفيفة الوزن

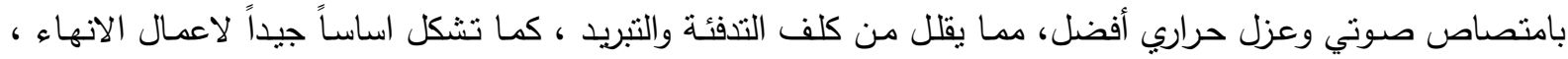
فضلا عن زيادة مقاومـة الانجماد والحريق(عدا الانواع العضوية القابلة للاشتعال ) ، وسـهولة القطع والتتقيب وتثتيـيت

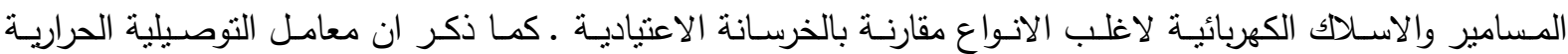

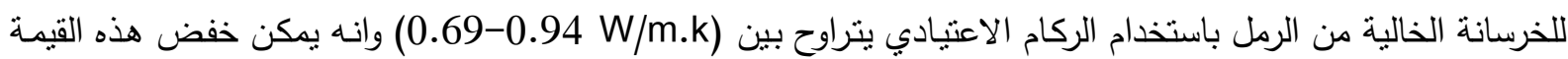

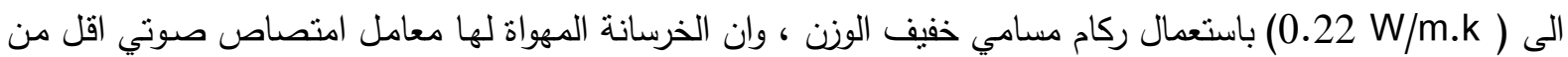

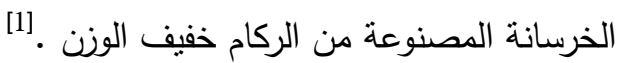
أعطى معهد الخرسانة الامريكي في (ACI 213R) تصنيف لخرسانة الركام خفيف الوزن ، كما انه عرف في

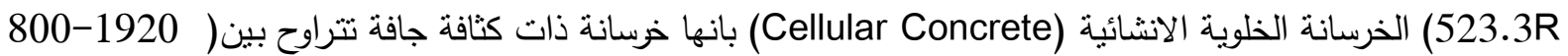

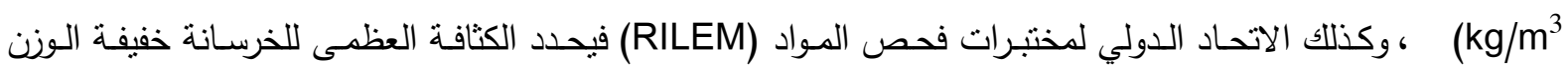
بـ (ASTM C330) ان الخرسانة الخفيفة الانشائية يجب ان لان لانتجاوز كتافتها الجافة

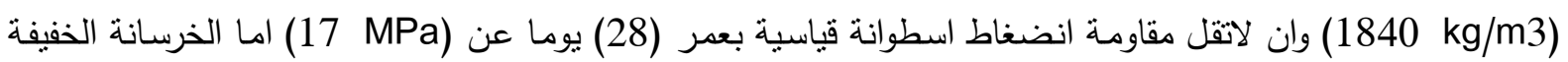

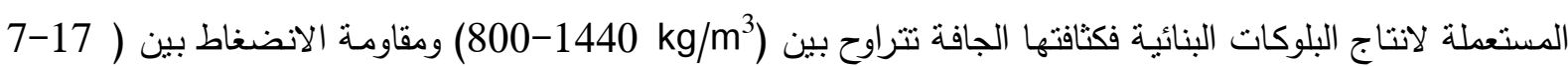

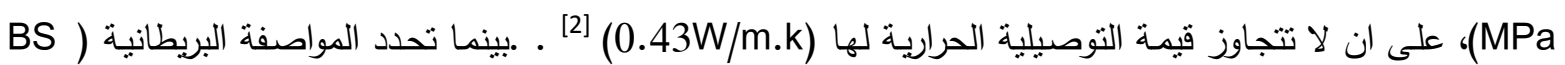

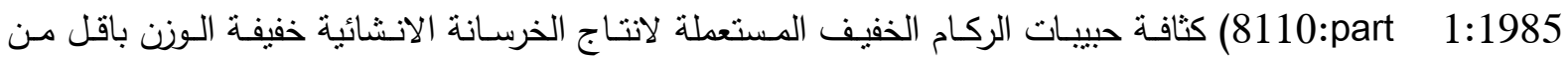

${ }^{[4]}\left(2000 \mathrm{~kg} / \mathrm{m}^{3}\right)$ امـا الباحث (T.Faust)حيث اشـار الى ان اعتبار الخرسانة التي تتراوح كثافتها بين (2200kg/m3-800) خرسانة انشائية خفيفة الوزن سيسمح بشمول كل انواع الخرسانة الخالية والواطئة الرمل (No-fines/Low-fines) بهذا التصنيف

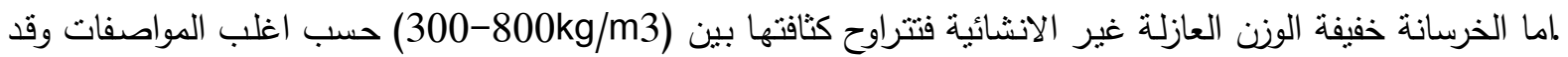
حددت (ASTM C 332) مقاومة انضغاط اسطوانة قياسية بعدر (28) يوم لهذه الخرسانة بين (0.7-7MPa) وان لاتزيد قيمة التوصيلية الحرارية لها عن (0.22 W/m.k) ولقد درس الباحث (Sagmeister) العزل الحراري لنماذج من الخرسانة الخالية من الرمل وفال ان التوصيلية الحراريـة تعتمد بالدرجة الاولى على كثافة الخرسانة حيث تزداد بزيادتها وبدرجة اقل على الخواص الحرارية للمجاميع المستعملة ، كما اثار الى ان قيم التوصيلية الحرارية للوحدات الانثائية تختلف عن قيمة التوصيلية الحرارية للجدار الذي تبنى منه وانهـ يجب الانتباه عند البنـاء لمنع تكون جسور لانتقال الحرارة خـلال سمك الجدار ، واضـاف ان للرطوبـة الممتصة من قبل لتصل

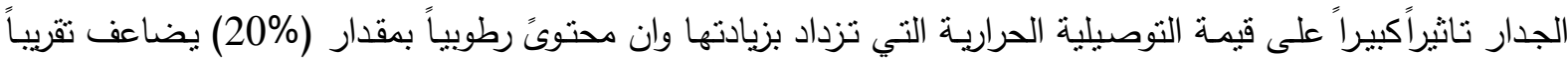

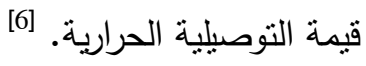

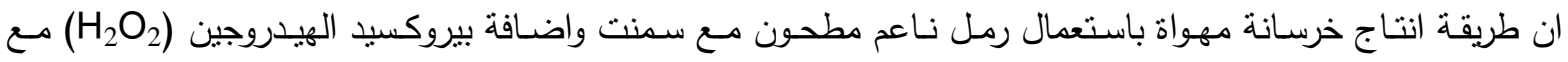
مسحوق القاصر (CaCl(OCl) ثم معالجتها بالمحمم عند (180 Co ) وضغط (10) جو لمدة (18) ساعة مما يسبب تقاعل السيليكا الموجودة في الرمل مع النورة الموجودة في الاسمنت مسبباً زيادة في مقاومة الانضغاط وتقليل انكماش بـاش [7] الجفاف للخرسانة الناتجة الخرسانة المهواة ، في هذا النوع من الخرسانة يتم تكوين مسامات هوائية ضمن كتلة مونة الخرسانة وذلك باحدى الطريقتين الاتينتين: أ.استعمال مواد كيميائية (مسحوق الألمنيوم او بإضـافة مادة بيروكسيد الهيدروجين مع المسحوق القاصر ) تولد فقاعات غازية ، وتسمى هذه الخرسانة بالخرسانة الغازية (Gas Concrete) . 
ب.اضافة رغوة منتجة بشكل مستقل الى الخرسانة فتسبب حدوث فقاعات هوائية ضمن كتلة الخرسانة ، وتسمى هذه الخرسـانة بالخرسـانة الرغويـة (Foamed Concrete)، ويفضل اسـتعمال ركام خفيف الـوزن كركسام الفيرميكولايــ (Permiculite) الخرسانة المهواة قد تحتوي ركاماً خشناً او قد لاتحتوي وغالباً مايكون الركام من النوع الخفيف ومن امتلة الخرسانة المهواة

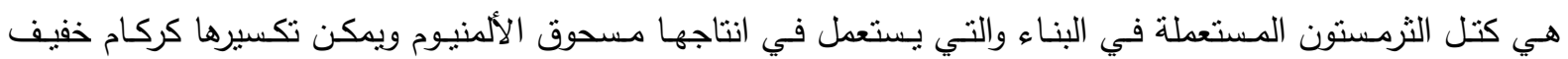

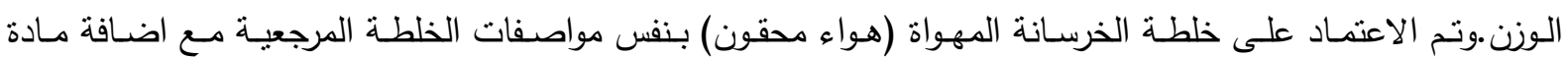
المسحوق القاصر (bleaching powder) (CaCl(OCl) (4) بنسبة (4\%) من وزن الاسمنت ثم اضيف ماء الخلط واخيرا" اضيفت مادة بيروكسيد الهيدروجين (H2O2) بنسبة (6\%) من وزن الاسمنت. ان تفاعل مادة المسحوق القاصر ومادة بيروكسيد الهيدروجين انتج الاوكسجين الحر والذي بدوره سبب حدوث فقاعات غازية داخل مونـة الخرسانة وكذللك الهواء

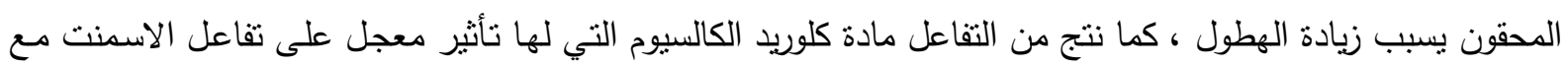
الماء وكما في المعادلة: - الة

$\mathrm{CaCl}(\mathrm{OCl})+\mathrm{H} 2 \mathrm{O} 2 \longrightarrow \mathrm{CaCl} 2+\mathrm{H} 2 \mathrm{O}+\mathrm{O} 2$

ان مقارنة انواع الخرسانة خفيفة الوزن تعتمد على المقارنة بين خصائصها المختلفة ومن اهمها الكثافة الجافة و مقاومـة

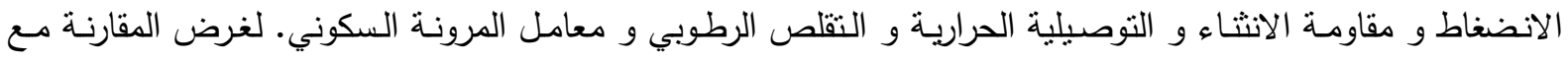
الخرسانة تم اختيار نوعين من الخرسانة خفيفة الوزن هما خرسانة الركام خفيف الوزن (باستعمال انواع مختلفة من الركام

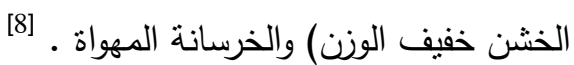
ان هذا النوع من الخرسانة (المهواة) تتكون فقاعات من الغازات والهواء في وسط الخرسانة وهي في حالة طازجة ويظل التركيب مسامي بعد التصلب ويمكن انتاجها بطريقتين اما انتاج غازات في الخلطة بتفاعلات كيمياوية ومن المواد الشائعة المولدة للغـازات مسحوق مـن بـودرة الالمنيوم او بـودرة الزنـك وعند الخلط مـع الاسـنت تتكون فقاعـات مـن

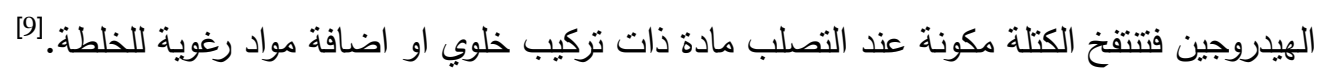
معامل التوصيل الحراري للخلطـات الخرسانية : يعتبر معامل التوصيل الحراري للكونكريت المقياس للتوصيل الحراري ويمكن تعرفه بانه عدد الوحدات الحرارية التي تمر خلال وحدة المساحة من المادة وبسمك يساوي وحدة واحدة وخلال وحدة زمنية واحدة عندما يكون الفرق ببن درجات الحرارة لوجهي الجسم درجة واحدة ويقاس بوحدة (W/m.k). حيث حددت الموصفات العالميـة طرقا عديدة لقياس الموصلية الحراريـة ومنها البريطانية والامريكية ، وفي هذا البحث اعتمدت معادلة (ACl 122R-02) التي تربط بين الكثافة الجافة ومعامل التوصيل الحراري لنماذج مجففة بالفرن وحسب المعادلة رقم (2) : (2) - (2) $K=0.072 \times e^{0.00125 p}$ (2) ${ }^{[3]}$

تمتلك خرسانة الركام خفيف الوزن مقاومة انضغاط و مقاومة انثاء و معامل مرونـة وتوصيلية حرارية اقل من الخرسانة

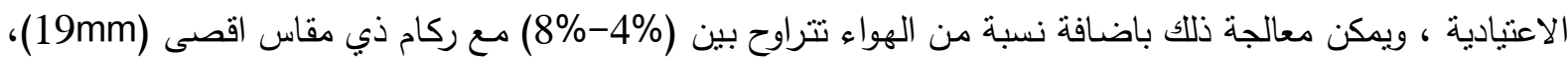

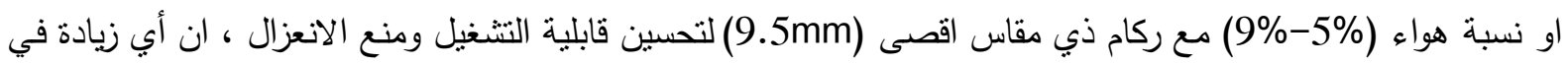
نسبة الهواء بمقدار (1\%) عن الحدود السابقة تسبب نقصان في المقاومـة الانضغاطية قدره (1\% MPa الخرسانة المهواة :هي خرسانة حاوية على فجوات صغيرة منتشرة ضمن كتلتها تانى من اضافة فقاعات غازية او هوائية 
الى الخرسانة الطرية. تمتاز الخرسانة المهواة بقابلية كبيرة على التمدد الحراري والانكماش ، ولها مقاومة عالية للحريق ،

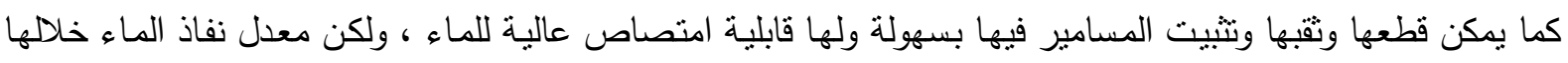
قليل لان فجواتها غير منصلة مما يعطيها مقاومة جيدة للانجماد، ومن المككن تحسين خواص الخرسانة المهواة بمعالجتها بالمحمم (Autoclave).

وكذلك تم دراسة العديد من خواص خرسانة البوليستايرين العازلة وتاثير نسبة البوليستارين الى الاسمنت على خواصها المختلفة وخاصة العزل الحراري والمعاوقة الصوتية (Acoustic Impedance).

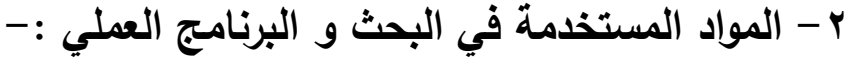

تم ذكر نوعية المواد المستخدمة في الخلطة الخرسانية وكذلك مقارنتها مـع المواصفات المعتمدة من قبل كل مادة

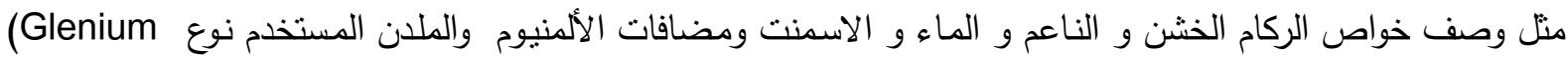

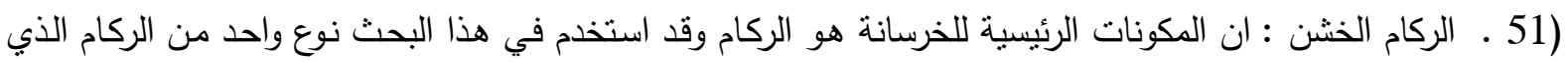
استخدم في مشاريع جامعة ديالى.ان نتائج الفحص التي اجريت في مختبر ديالى الانشائي على عينات من هذا لردان الركام

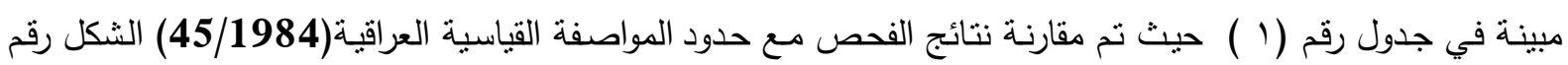
(1) يبين التحليل المختبري لهذا النوع من الركام. الركام الناعم : يتم تجهيز هذا النوع من الركام من أحد المقالع الذي يقع شمال محافظة ديالى (مقلع الصدور) وان نتائج

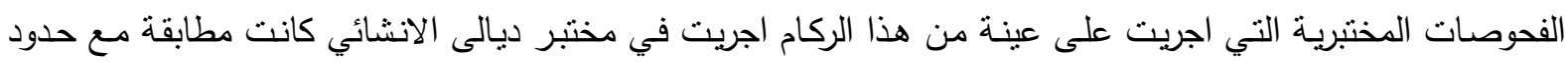

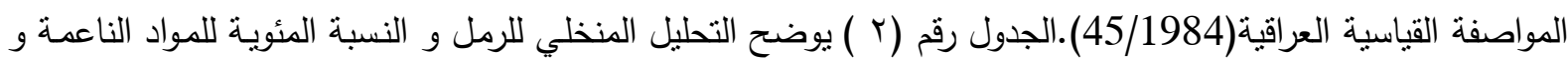
الكبريتات و الشكل رقم (Y) يبين التحليل المنخلي لهذا النوع من الركام . الاسمنت :تم اختيار اسمنت نوع ( طاسلوجة ) و هو سمنت بورتلاندي اعتيادي ذو المواصفات مبينة في جدول رقم (ع). مـاء الخلط :ان المـاء المستخدم لعمل الخلطات الخرسانية التجريبية هو الماء الصالح للشرب كما استخدم نفس المـاء للمعالجة ايضاً. النماذج:استخدمت نماذج مكعبات(15cmx15cmx15cm) للخلطة بدون المضاف (الخلطة المرجعية) وتمت المقارنـة

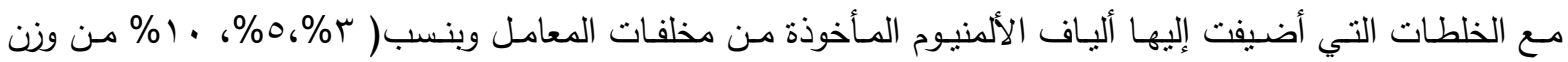
الاسمنت) • تم العمل بطريقة التصميم للخطة الأمريكية المبينة في الجدول رقم ( ب ).بعد الخلط الميكانيكي الجيد تم

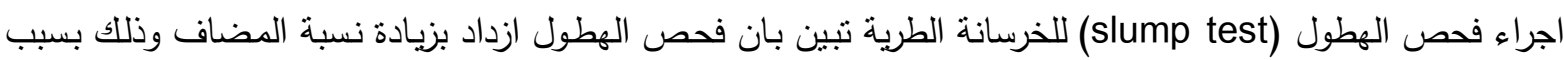

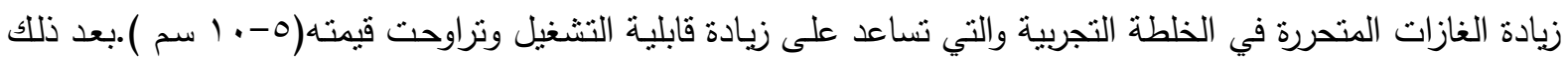
تم صبت النمـاذج وفق الموصفات في نقس الوقت وتركت في احواض المعالجـة لحين موعد الفحص(فحص الكثافـة ومقاومة الانضغاط )هوتم اخذ ثلاثة نماذج لكل نسبة وكذللك لكل عمر فحص.

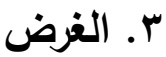

ان الغرض من هذا البحث هو لمعرفة مدى تاثير اضافة الياف الالمنيوم الماخوذة من مخلفات معامل الالمنيوم على معامل التوصيل الحراري للخرسانة بصورة رئيسية بالاضـافة الى تاثير ذلك على مقاومة الانضغاط ،مما يتيح انتاج خرسانة ذات معامل توصيل حراري واطئ. 


\section{ع .المناقشة و النتائج}

لغرض دراسة تأثثر اضافة الياف الالمنيوم المأخوذة من مخلفات معامل الالمنيوم على معامل التوصيل الحراري للخرسانة مع الاخذ بنظر الاعنبار مدى ناثر مقاومة الانضغاط بهذا النوع من المضافات اجريت عدة فحوصات لمكعبات خرسانية قياسية (15cmx15cmx15cm) وحسب المواصفات لقياسية المعتمدة لكل فحص. بعد ما تم صب خلطة مرجعية لغرض المقارنة مـع باقي الخلطات التي تحتوي على مضاف الالمنيوم لثلاث نسب هي بـ من من وزن الاسمنت

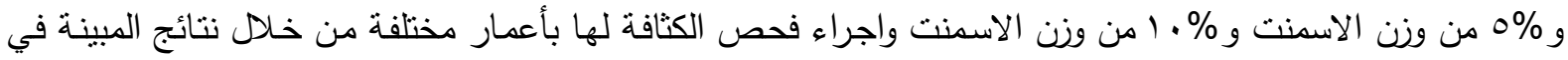

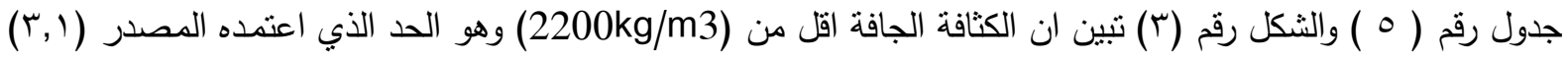

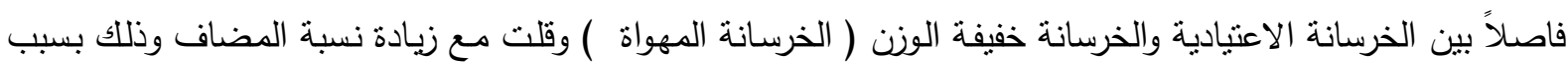

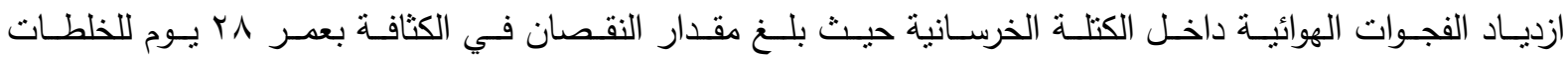

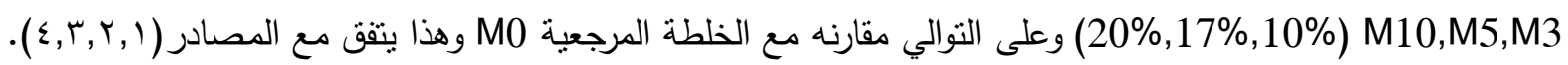
وهذا بدوره يوثر على معامل التوصيل الحراري حيث يقل مع زيادة نسبة المضاف لان له ارتباط وثيق مـع الكثافة كما مبين في الثكل رقم (0)، وحسب معادلة الكود الامريكي(ACI 122R) ، والجدول رقم (0) يتضمن قيم التوصيلية الحرارية

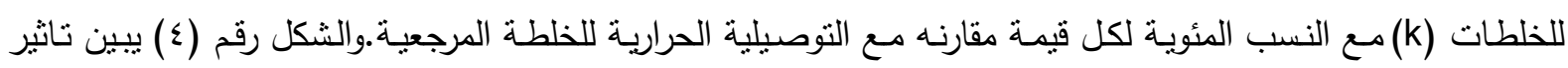

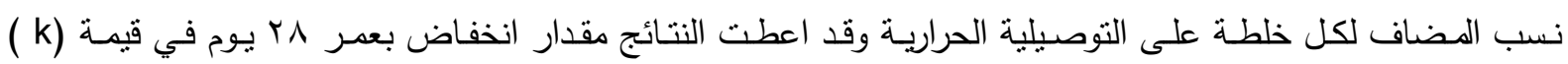
للخلطات M10,M5,M3

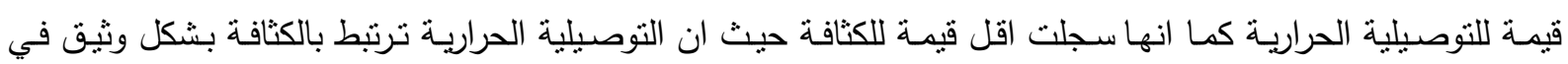
الخرسانة خفيفة الوزن، لان طبيعتها الخلوية تسبب وجود نسبة كبيرة من الفراغات الهوائية ضمن هيكلها، والهواء الساكن

يعتبر من احسن العوازل للحرارة، لذلك فان انخفاض الكثافة سيصاحبه انخفاض في قيمة التوصيلية الحرارية . وفحص مقاومة الانضغاط يتضمن الجدول رقم (0) والشكل رقم (؟) نتائج فحص مقاومة الانضغاط للخلطات الخرسانية

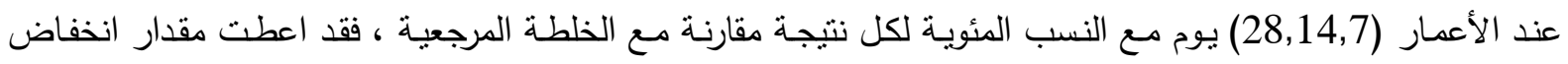
نسبة تراوحت بين (30\%-54\%) من مقاومة الخلطة المرجعية، حيث ان فنل نقاط الاتصال(28) (Points of Contact)

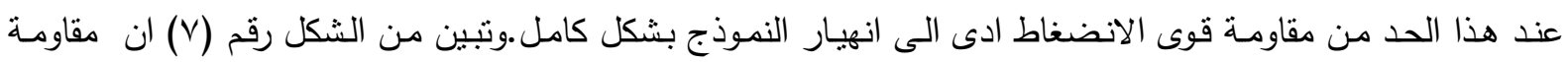

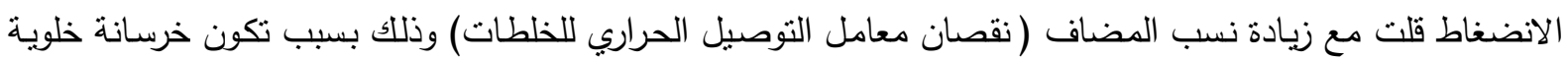
داخل الكتلة الخرسانية سببها تحرر الغازات من تفاعل الالمنيوم مع مركبات الاسمنت.

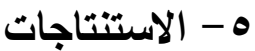

ا ـان لنسبة المضاف من وتن الاسـنت تاثيراً مباشراً على خصائص الخرسـانة الطريـة والمتصلبة، فالنماذج التي اعدت باستعمال نسب مختلفة من المضاف ازدادت قيمة الهطول (5-10) سم مع زيادة النسبة .

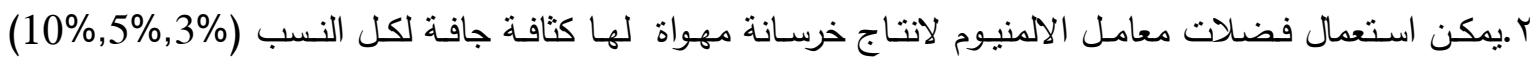
واعمار الفحص(28,14,7) يوم ، بحدود (93\% - (80\%) اقل من الكثافة الجافة للخرسانة الاعتياديه وبالتتابع. ب بمكن انتاج خرسانة مهواة وذات معامل توصيل حراري واطئ من خلال إضافة نسبة من مضاف الالمنيوم للخرسانة

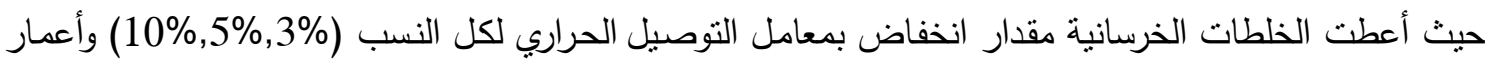
الفحص(28,14,7) يوم تراوح مابين (17\%-45\%) اقل من الخلطات المرجعية . 


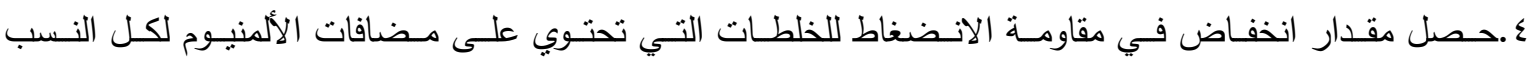

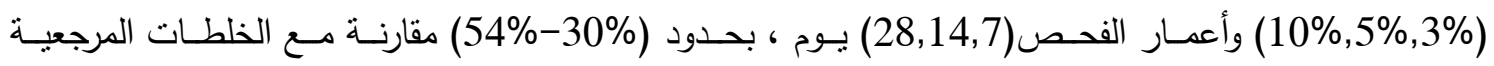

• وبالتتابع

- -7

1. Neville A.M. "Properties of Concrete". Third edition, Pitman Publishing Limited, London, UK, 1981.

2. "Guide for Structural Lightweight Aggregate Concrete". ACI 213R-03, American

Concrete Institute, Detroit, Michigan. 2003.

3. "Guide to Thermal Properties of Concrete and Masonry Systems",ACI 122R-02, American Concrete Institute, Detroit, Michigan. 2002.

4. British Standards Institute, B.S 8110: part 1: 1985 "Structural Use of Concrete-Code of Practice for Design and Construction".

5. Faust T. "Supplements to MC-90 for Lightweight Aggregate Concrete", Lacer, No.5, 2000,pp 75-84.

6. Sagmeister B. " Heat-Insulating from No-Fines Lightweight Concrete", available via internet at the web site:

http://www.baustoffchemie.de/en/db/lightweight-concrete/ ,2001.

7. Shrivastava O.P. "Lightweight Aerated or Cellular Concrete-a Review", The Indian Concrete Journal, Vol.51, January, 1977, pp18-23.

8. Hassan I.M."Comparative Study of Some Properties of Lightweight Concrete " MSc.Thesis of the College of Engineering-University of Mosul ,July,2004

9. Mahmmod. I., A. Abdel-Reheem, Y. Abou-Mosallam, and A. Shihata, "Acoustic and Thermal Insulation of Lightweight Concrete" Eighth Arab Structural Engineering Conference, Faculty of Engineering, Cairo University, 2000, pp 1559-1569.

• . . مؤيد نـوري الخلف، هنـاء عبد يوسف " تكنولوجيـا الخرسـانة ". الطبعـة الاولى، الجامعـة التكنولوجيـة، بغداد،

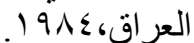

ال. ندى مهدي فوزي الجيلاوي "خو اص الخرسانة خفيفة الوزن بالاشـارة الى العزل الحراري والمعاوقة الصوتية".

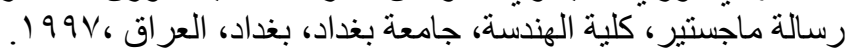




$$
\text { جدول (1): تدرج الركام الخشن. }
$$

\begin{tabular}{|c|c|c|}
\hline $\begin{array}{c}\text { Sieve size } \\
(\mathrm{mm})\end{array}$ & $\begin{array}{c}\text { \% Passing by } \\
\text { Weight }\end{array}$ & $\begin{array}{c}\text { Limits of the Iraqi specification } \\
\text { No.45/1984 }\end{array}$ \\
\hline 12.5 & 100 & 100 \\
\hline 10 & 88.6 & $85-100$ \\
\hline 5 & 10.8 & $0-25$ \\
\hline 2.36 & 0 & $0-5$ \\
\hline
\end{tabular}

$$
\text { جدول (ץ): تدرج الركام الناعم. }
$$

\begin{tabular}{|c|c|c|}
\hline $\begin{array}{c}\text { Sieve size } \\
(\mathrm{mm})\end{array}$ & $\begin{array}{c}\text { \% Passing by } \\
\text { Weight }\end{array}$ & $\begin{array}{c}\text { Limits of the Iraqi specification } \\
\text { No.45/1984 (zone 2) }\end{array}$ \\
\hline 4.75 & 100 & $90-100$ \\
\hline 2.36 & 93.3 & $75-100$ \\
\hline 1.18 & 84.0 & $55-90$ \\
\hline 0.60 & 57.2 & $35-59$ \\
\hline 0.30 & 27.5 & $0-10$ \\
\hline 0.15 & 8 & \\
\hline
\end{tabular}

جدول ( ץ ): الخلطة التصميمية المستخدمة في البحث.

\begin{tabular}{|c|c|c|c|c|c|c|c|}
\hline $\begin{array}{c}\text { No. of } \\
\mathrm{mix}\end{array}$ & $\begin{array}{c}\text { Cement } \\
\mathrm{kg} / \mathrm{m}^{3}\end{array}$ & $\mathrm{~W} / \mathrm{C}$ & $\begin{array}{c}\text { Coarse } \\
\mathrm{gg} . \\
\mathrm{kg} / \mathrm{m}^{3}\end{array}$ & $\begin{array}{c}\text { Fine agg kg/ } \\
\mathrm{m}^{3}\end{array}$ & $\begin{array}{c}\text { Water } \mathrm{kg} / \\
\mathrm{m}^{3}\end{array}$ & $\begin{array}{c}\text { Superplastizer } \\
\%\end{array}$ & $\begin{array}{c}\text { Aluminum } \\
\%\end{array}$ \\
\hline $\mathrm{M} 0 \%$ & 440 & 0.35 & 1050 & 760 & 154 & 1 & ---- \\
\hline $\mathrm{M} 3 \%$ & 440 & 0.35 & 1050 & 760 & 154 & 1 & 3 \\
\hline $\mathrm{M} 5 \%$ & 440 & 0.35 & 1050 & 760 & 154 & 1 & 5 \\
\hline $\mathrm{M} 10 \%$ & 440 & 0.35 & 1050 & 760 & 154 & 1 & 10 \\
\hline
\end{tabular}




$$
\text { جدول (؛ ): التركيب الكيميائي للاسمنت المستخدم في البحث. }
$$

\begin{tabular}{|c|c|c|c|}
\hline Oxide composition & Abbreviation & Content (percent) & $\begin{array}{c}\text { Limit of Iraqi } \\
\text { specification } \\
\text { No.5/1984 }\end{array}$ \\
\hline Lime & $\mathrm{CaO}$ & 63.19 & --- \\
\hline Silica & $\mathrm{SiO}_{2}$ & 20.60 & --- \\
\hline Alumina & $\mathrm{AL}_{2} \mathrm{O}_{3}$ & 4.10 & --- \\
\hline Iron Oxide & $\mathrm{Fe}_{2} \mathrm{O}_{3}$ & 4.48 & --- \\
\hline Sulphate & $\mathrm{SO}_{3}$ & 1.98 & $<2.8 \%$ \\
\hline Magnesia & $\mathrm{MgO}$ & 2.28 & $\leq 5 \%$ \\
\hline Loss on Ignition & L.O.I & 2.45 & $\leq 4 \%$ \\
\hline Insoluble residue & I.R & 0.47 & $\leq 1.5 \%$ \\
\hline Lime saturation factor & L.S.F & 0.94 & $0.66-1.02$ \\
\hline $\begin{array}{c}\text { Main compounds (Bogue's } \\
\text { equations) }\end{array}$ & & & \\
\hline Tricalcium Silicate & $\mathrm{C}_{3} \mathrm{~S}$ & 57.11 & \\
\hline Di Calcium Silicate & $\mathrm{C}_{2} \mathrm{~S}$ & 16.23 & \\
\hline Tri Calcium Aluminate & $\mathrm{C}_{3} \mathrm{~A}$ & 8.39 & $>5 \%$ \\
\hline Tetra Calcium Alumina Ferrite & $\mathrm{C}_{4} \mathrm{AF}$ & 13.62 & \\
\hline
\end{tabular}


جدول(ه): نتائج فحوصات الخلطات الخرسانية التجريبية بأعمارها ونسبها المختلفة.

\begin{tabular}{|c|c|c|c|c|c|c|c|c|}
\hline $\begin{array}{l}\text { Average } \\
\text { sample } \\
\text { of mix }\end{array}$ & Age & $\begin{array}{c}\text { Aluminum } \\
\%\end{array}$ & $\begin{array}{c}\text { Density } \\
\mathrm{kg} / \mathrm{m} 3\end{array}$ & $\begin{array}{c}\text { Reduction } \\
\text { Density } \\
\%\end{array}$ & $\begin{array}{c}\text { Thermal } \\
\text { conductivity } \\
\text { (w/m.k) }\end{array}$ & $\begin{array}{c}\text { Reduction } \\
\text { Thermal } \\
\%\end{array}$ & $\begin{array}{c}\text { compressive } \\
\text { Strength } \\
\text { (MPa) }\end{array}$ & $\begin{array}{l}\text { Reduction } \\
\text { compressive } \\
\text { Strength \% }\end{array}$ \\
\hline M 0\% & 7 & ------ & 2250 & 100 & 1.2 & 100 & 19.8 & 100 \\
\hline M3\% & 7 & 3 & 2100 & 7 & 0.99 & 17 & 13.85 & 30 \\
\hline M 5\% & 7 & 5 & 1910 & 15 & 0.78 & 35 & 11.4 & 42 \\
\hline M 10\% & 7 & 10 & 1830 & 19 & 0.71 & 41 & 10.12 & 49 \\
\hline M $0 \%$ & 14 & ------- & 2350 & 100 & 1.36 & 100 & 26.66 & 100 \\
\hline M $3 \%$ & 14 & 3 & 2130 & 9 & 1.03 & 24 & 15.3 & 43 \\
\hline M 5\% & 14 & 5 & 1950 & 17 & 0.82 & 39 & 13.32 & 50 \\
\hline M 10\% & 14 & 10 & 1881 & 20 & 0.76 & 44 & 12.47 & 53 \\
\hline M $0 \%$ & 28 & ------- & 2398 & 100 & 1.44 & 100 & 32.75 & 100 \\
\hline M $3 \%$ & 28 & 3 & 2150 & 10 & 1.06 & 27 & 17.54 & 46 \\
\hline M 5\% & 28 & 5 & 1985 & 17 & 0.86 & 40 & 16.24 & 50 \\
\hline M $10 \%$ & 28 & 10 & 1920 & 20 & 0.79 & 45 & 15.16 & 54 \\
\hline
\end{tabular}




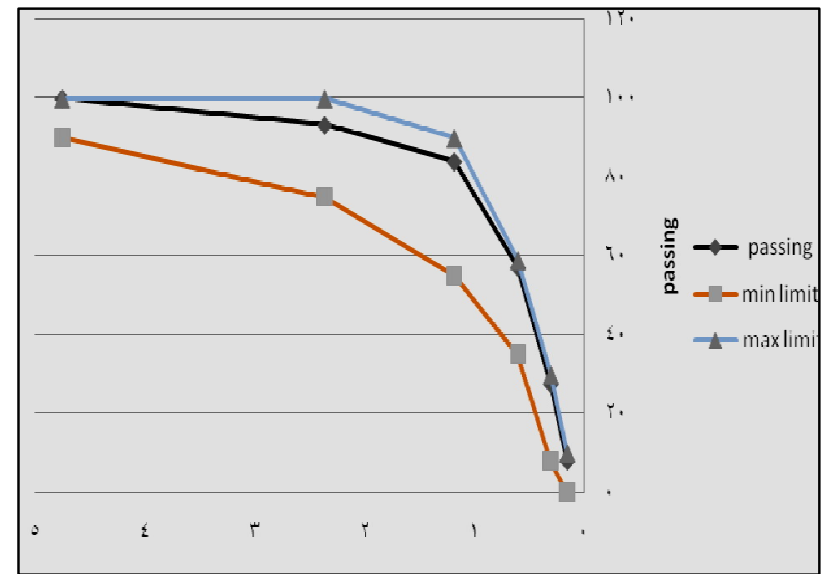

شكل (ץ): ت تدرج الركام الناعم.

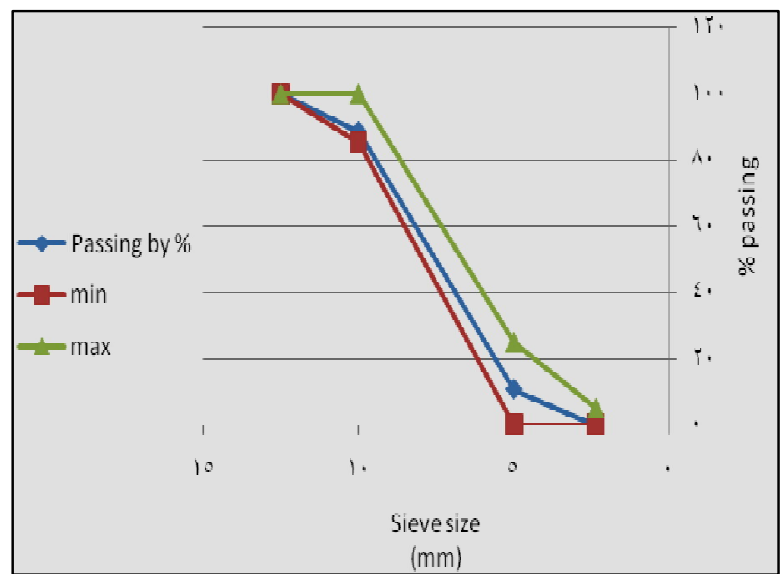

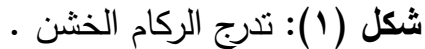

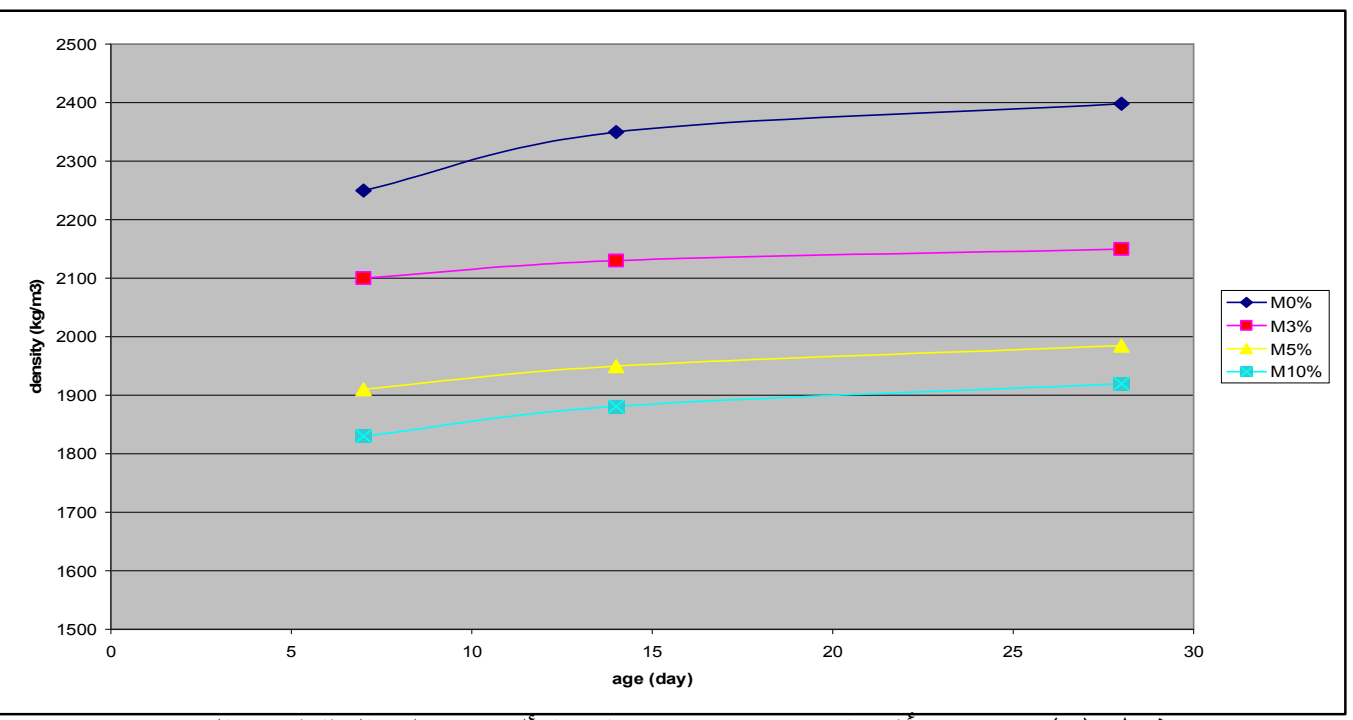

شكل (ץ): يوضح تأثثر اختلاف نسب مضاف الألمنيوم على الكثافة مع العمر •

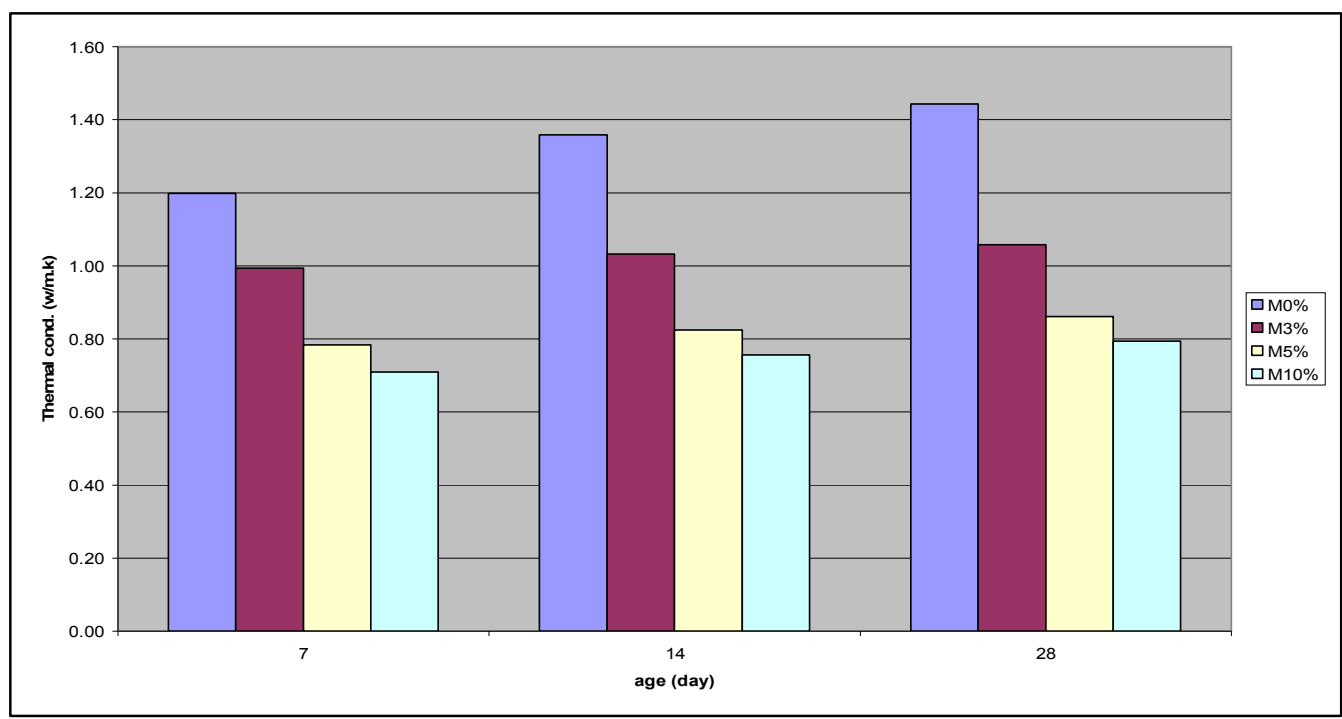

شكل (؛): يوضح نأثير اختلاف نسب مضاف الأمنيوم على معامل التوصيل الحراري مع العمر. 


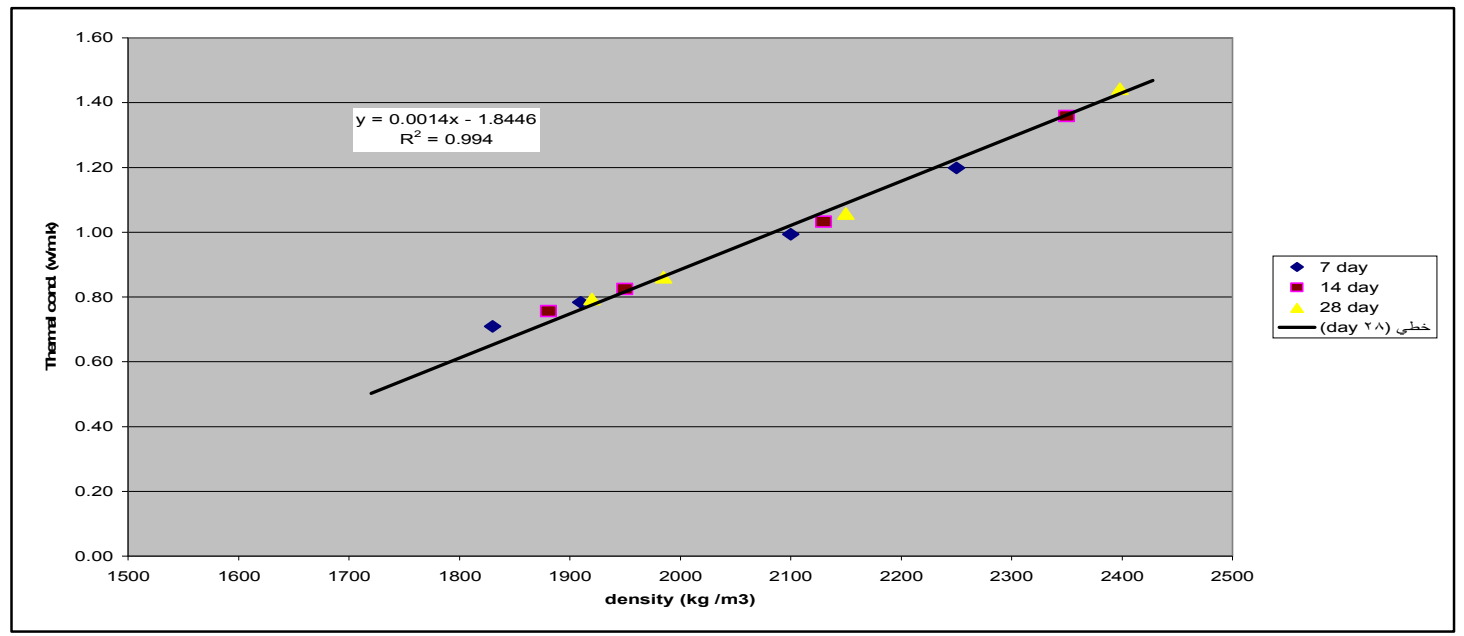

شكل (0): يوضح العلافة بين معامل التوصيل الحراري والكثافة لخلطات مختلفة من نسب الألمنيوم.

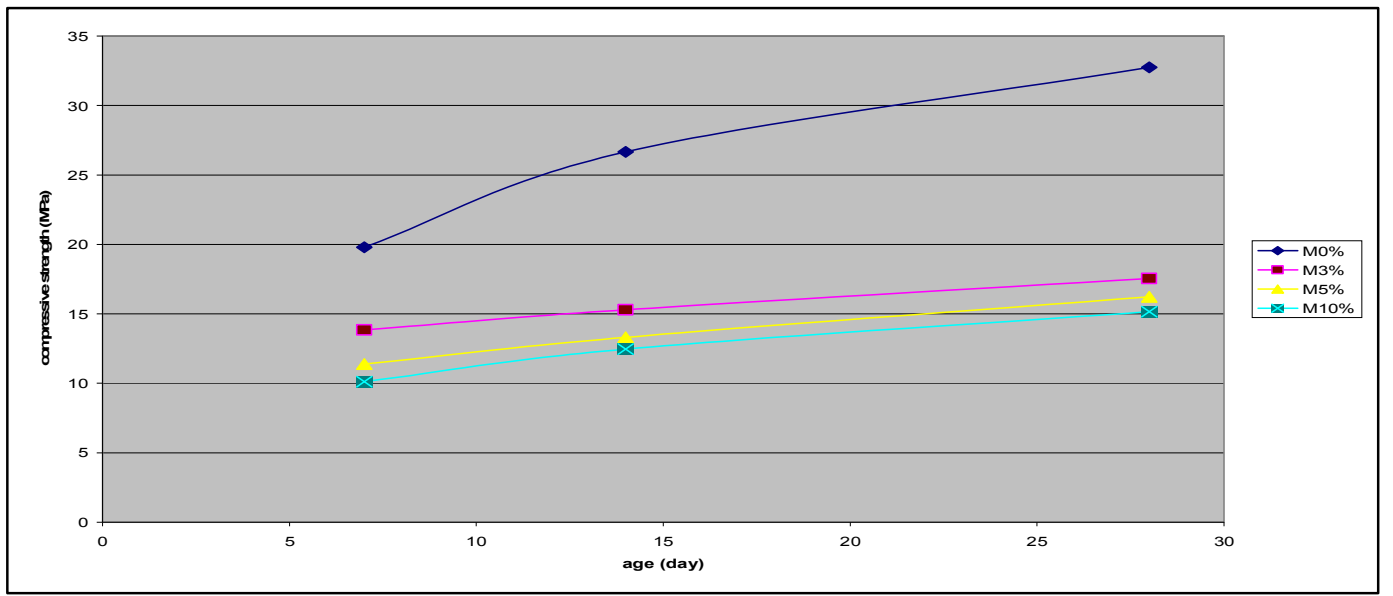

شكل (†): يوضح نأثنر اختلاف نسب مضاف الألمنيوم على مقاومة الانضغاط مع العمر.

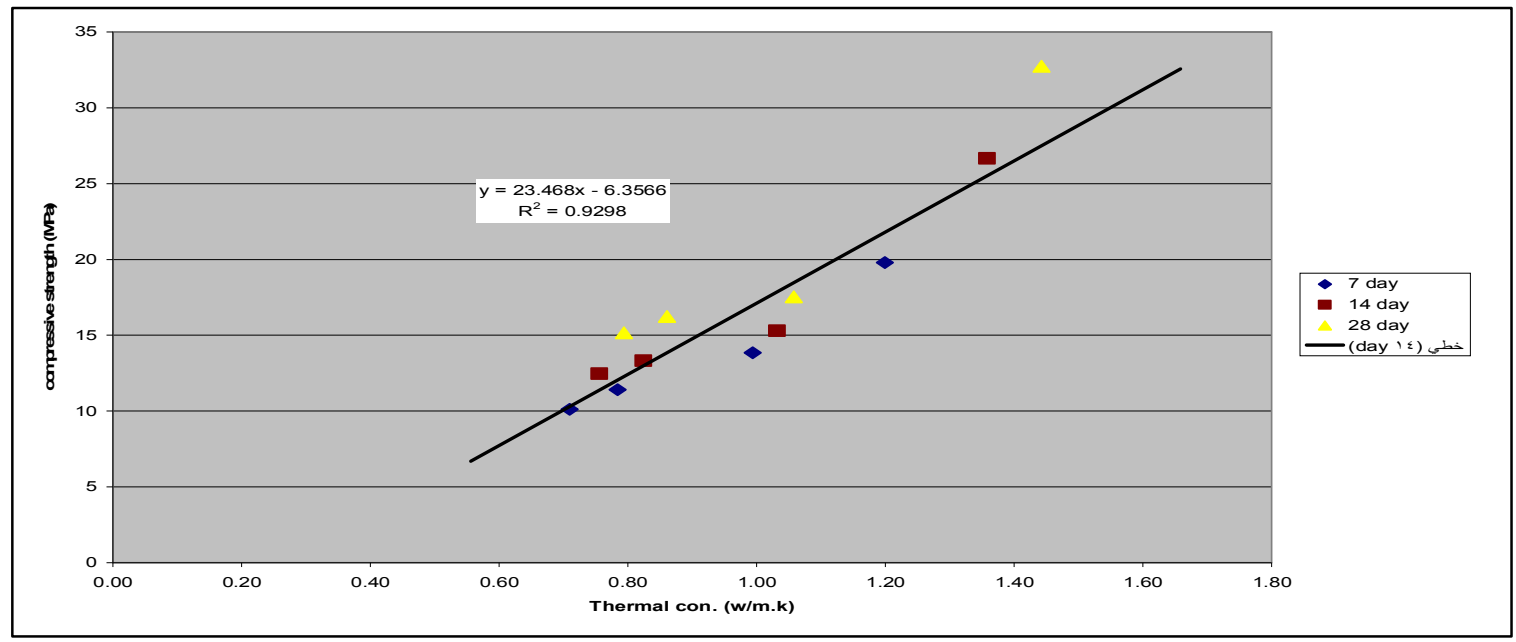

شكل (V): يوضح العلاقة بين مقاومة الانضغاط و معامل التوصيل الحراري لخلطات مختلفة من نسب مضاف الألمنيوم بأعمار مختلفة. 


\title{
EFFECT OF ADDING THE WASTE PRODUCTEDALUMINUM FACTORIES ON THE THERMAL CONDUCTIVITY FACTOR OF THE CONCRETE
}

\author{
Mohammed K. Albayti \\ Dr. Lecture \\ Ali H. Hammeed \\ Lecturer \\ Assal T. Hussein \\ Lecturer \\ College of engineering College of engineering \\ Baghdad University Diyala University \\ College of engineering \\ Diyala University
}

\begin{abstract}
This research was studying the effect of added aluminum fiber from the waste product of aluminum factories on the properties of concrete mixes. The study involved a reference concrete mix (M0) without admixture of aluminum and compare with three mixes (M3,M5, and M10), with three percent (3\%,5\%, and10\%) from weight of cement, Specifically The thermal conductivity factor $(\mathrm{k})$ was calculated depending on the equation of ACI code (122-R-2002) for mix concrete. The laboratory testes (slump flow, density, compressive strength) were doing for the cubes samples with dimension $(150 * 150 * 150)$ to concrete mixes(M0,M3,M5), and M10) in age (7,14,and 28)day. The affect thermal conductivity factor of concrete was depended on the effect of workability, density and compressive strength with age sample. The results were getting from the testing found the density, thermal conductivity factor and compressive strength decreasing with adding the admixture of aluminum (7\%$20 \%),(17 \%-45 \%)$ and $(30 \%-54 \%)$ respectively, because of the reaction between the aluminum fiber with compound of cement cause the babble gas (Cellular concrete) due to reduction in the density and compressive strength .
\end{abstract}

Keyword: Cellular concrete, thermal conductivity factor, admixture of aluminum, compressive strength, density . 